\title{
Improved Multiuser Detection in Asynchronous Flat- Fading Non-Gaussian Channels
}

\author{
K. Vidyullatha, Student Member, IEEE, \\ Electronics and Communication Engineering Department, \\ Kakatiya Institute of Technology and Science, \\ Warangal, Andhra Pradesh, India - 506105. \\ e-mail: v_kanchanapally@yahoo.com \\ V. Harish, Student Member, IEEE, \\ Electronics and Communication Engineering Department, \\ Kakatiya Institute of Technology and Science, Warangal, \\ Andhra Pradesh, India - 506015. \\ e-mail: harishvictory@gmail.com
}

\author{
S. V. N. L. Tejaswi, \\ Electronics and Communication Engineering Department, \\ Kakatiya Institute of Technology and Science, Warangal, \\ Andhra Pradesh, India - 506015. \\ e-mail: svnlchunduriece@gmail.com \\ T. Anil Kumar, Member, IEEE \\ Electronics and Communication Engineering Department, \\ RRS College of Engineering and Technology, Patancheru, \\ Andhra Pradesh, India - 502300. \\ e-mail: tvakumar2000@yahoo.co.in
}

Abstract - In this paper, a new M-estimator based multiuser detection in asynchronous flat-fading non-Gaussian CDMA channels is considered. A new closed-form expression is derived for the characteristic function of the multiple-access interference signals. Simulation results are provided to prove the effectiveness of the derived bit-error probabilities obtained with this expression in asynchronous flat-fading non-Gaussian CDMA channels.

Keywords- bit-error rate; code-division multiple-access; inter symbol interference; multiuser detection; M-estimation.

\section{INTRODUCTION}

Recently, the problem of robust multiuser detection in nonGaussian channels has been addressed in the literature [1] \& [2], which were developed based on the Huber and Hampel estimators. The Huber's estimator [3] can limit the effect of gross errors; however, the effect may still have to be large enough to reach an unacceptable level. The Hampel's estimator [4] avoids this problem by setting up three regions to reflect the influence of gross errors with different magnitudes. It is noticed that big gross errors greater than a given threshold will have no effect on the solution as a sharp rejection point may corrupt the estimation when observations are not carefully treated due to low redundancy. Hence, a new $M$ estimator is proposed for multiuser detection in asynchronous flat-fading non-Guassian channels in this paper. Further, a new closed-form expression is derived for computing the average biterror rate (BER) under asynchronous transmission conditions in flat-fading non-Gaussian channels using the characteristic function method.

\section{SYSTEM MODEL}

Consider the signal model of [5] in matrix notation, which can be written as

$$
\boldsymbol{y}(i)=\boldsymbol{A} \boldsymbol{\theta}(i)+\boldsymbol{n}(i)
$$

where $\quad \boldsymbol{y}(i) \cong\left[y_{1}(i), \ldots \ldots ., y_{N}(i)\right]^{T} \quad, \quad \boldsymbol{n}(i) \cong\left[n_{1}(i), \ldots \ldots ., n_{N}(i)\right]^{T}$, $\boldsymbol{\theta}(i) \cong(1 / \sqrt{N})\left[b_{1}(i) g_{1}(i), \ldots \ldots, b_{L}(i) g_{L}(i)\right]^{T}, \quad \boldsymbol{A} \equiv\left[\boldsymbol{a}_{1}, \boldsymbol{a}_{2}, \ldots ., \boldsymbol{a}_{L}\right]$, $\boldsymbol{a}_{l}=\left[a_{1}^{l}, \ldots \ldots ., a_{N}^{l}\right]^{T}, b_{l}(i)$ denote symbol stream for the $l$ th user, and $g_{l}(i)$ is the $l$ th channel fading coefficient $(N$ is the processing gain and $L$ is the number of active users). It is assumed that the sequence of noise samples $\{\boldsymbol{n}(i)\}$ is a sequence of independent and identically distributed (i.i.d.) complex random variables whose in-phase and quadrature components are independent non-Gaussian random variables with a common probability density function (pdf) $f$. The pdf of this noise model has the form

$$
f=(1-\varepsilon) \aleph\left(0, v^{2}\right)+\varepsilon \aleph\left(0, \kappa v^{2}\right)
$$

with $v>0,0 \leq \varepsilon \leq 1$, and $\kappa \geq 1$. Here $\aleph\left(0, v^{2}\right)$ represents the nominal background noise and the $\aleph\left(0, \kappa v^{2}\right)$ represents an impulsive component, with $\varepsilon$ representing the probability that impulses occur.

\section{BER ANALYSIS}

In this section, the characteristic function method is used to compute the average BER under asynchronous transmission conditions in flat-fading non-Gaussian channels. We first examine the statistics of each interferer. This analysis parallels that of [6], and so the presentation here is brief. We have the definition of interference from each interferer as $I_{k}=G_{k} W_{k}$, where $G_{k}$ is a zero-mean unit-variance guassian random variable and $W_{k}$ is defined in terms of random variables $P_{k}, Q_{k}, X_{k}, Y_{k}, S_{k}$ as

$$
W_{k}=P_{k} S_{k}+Q_{k}\left(1-S_{k}\right)+X_{k}+Y_{k}\left(1-2 S_{k}\right)
$$


where, $S_{k}$ is a uniform random variable over [0,1), $P_{k}$ and $Q_{k}$ are symmetric Bernoulli random variables, $X_{k}$ and $Y_{k}$ are the discrete random variables that represent the sums of different sets of independent symmetric Bernoulli random variables. Thus, given $W_{k}, I_{k}$ is a Gaussian random variable with zero-mean and conditional variance $\sigma_{I_{k} \mid W_{k}}^{2}=W_{k}^{2}$. This implies through (3) that $I_{k}$ given $P_{k}, Q_{k}, X_{k}, Y_{k}, S_{k}, B$ (where $B$ equals the number of chip boundaries with transitions in target user's signature waveform) is Gaussian and the conditional pdf for $I_{k}$ follows as

$$
\begin{aligned}
& f_{I_{k} \mid P_{k}, Q_{k}, X_{k}, Y_{k}, S_{k}, B}\left(i_{k}\right) \\
& =\frac{1}{\sqrt{2 \pi}\left|P_{k} S_{k}+Q_{k}\left(1-S_{k}\right)+X_{k}+Y_{k}\left(1-2 S_{k}\right)\right|} \\
& \times \exp \left\{-\frac{i_{k}^{2}}{2\left[P_{k} S_{k}+Q\left(1-S_{k}\right)+X_{k}+Y_{k}\left(1-2 S_{k}\right)\right]^{2}}\right\}
\end{aligned}
$$

since $W_{k}$ may take negative values, a modulus operation is required in (3). Averaging over $P_{k}, Q_{k}, X_{k}, Y_{k}$ (which is equivalent to averaging over all interferers, spreading sequences and data sequences), yields

$$
\begin{aligned}
& f_{I_{k} \mid S_{k}, B}\left(i_{k}\right) \\
& =\frac{1}{4 \sqrt{2 \pi}} 2^{-(N-1)} \sum_{i \in \mathbf{A}} \sum_{j \in \mathbf{B}}\left(\begin{array}{c}
A \\
\frac{i+A}{2}
\end{array}\right)\left(\begin{array}{c}
B \\
\frac{j+B}{2}
\end{array}\right) \\
& \quad \times\left\{\sum_{l=1,2,3,4} \frac{1}{\sigma_{l}\left(i, j, S_{k}\right)} \exp \left\{-\frac{i_{k}^{2}}{2 \sigma_{l}^{2}\left(i, j, S_{k}\right)}\right\}\right\}
\end{aligned}
$$

where,

$$
\begin{gathered}
\sigma_{1}^{2}\left(i, j, S_{k}\right)=\left[1+i+j\left(1-2 S_{k}\right)\right]^{2} \\
\sigma_{2}^{2}\left(i, j, S_{k}\right)=\left[2 S_{k}-1+i+j\left(1-2 S_{k}\right)\right]^{2} \\
\sigma_{3}^{2}\left(i, j, S_{k}\right)=\left[1-2 S_{k}+i+j\left(1-2 S_{k}\right)\right]^{2} \\
\sigma_{4}^{2}\left(i, j, S_{k}\right)=\left[-1+i+j\left(1-2 S_{k}\right)\right]^{2}
\end{gathered}
$$

note that to obtain (6), it was considered that $X_{k}$ and $Y_{k}$ given $B$ are independent. It is clear from (6) that the pdf of $I_{k}$ given $S_{k}$ and $B$ is not gaussian, though the functional form is a weighted summation of "Gaussian-like" terms. We postpone averaging $S_{k}$ here since they appear in the denominators of the exponential function arguments giving an intractable integral. The characteristic function of $I_{k}$, given $S_{k}$ and $B$, is

$$
\begin{aligned}
\Phi_{I_{k} \mid S_{k}, B}(\omega) & =\frac{2^{-(N-1)}}{4} \sum_{i \in A} \sum_{j \in B}\left(\begin{array}{c}
A \\
\frac{i+A}{2}
\end{array}\right)\left(\begin{array}{c}
B \\
\frac{j+B}{2}
\end{array}\right) \\
& \times\left\{\sum_{l=1,2,3,4} \exp \left\{-\frac{1}{2} \sigma_{l}^{2}\left(i, j, S_{k}\right) \omega^{2}\right\}\right\}
\end{aligned}
$$

The $S_{k}$ 's now appear in the numerators of the exponential function arguments and averaging can be carried out to give

$$
\begin{aligned}
& \begin{aligned}
\Phi_{I_{k} \mid B}(\omega) & =\int_{0}^{1} \Phi_{I_{k} \mid S_{k}, B}(\omega) d S_{k} \\
& =\frac{2^{-(N-1)}}{4} \sum_{i \in A} \sum_{j \in B}\left(\begin{array}{c}
A \\
\frac{i+A}{2}
\end{array}\right)\left(\begin{array}{c}
B \\
\frac{j+B}{2}
\end{array}\right)
\end{aligned} \\
& \times[J(i+1, j)+J(i, j-1)+J(i, j+1)+J(i-1, j)]
\end{aligned}
$$

where,

$$
J(i, j)=\left\{\begin{array}{cc}
\left.\left.\frac{\sqrt{\pi / 2}}{j \mid \omega}\{Q|\omega|(i-j))-Q \mid \omega(i+j)\right)\right\}, & (j, \omega \neq 0) \\
\exp \left(-\frac{1}{2} i^{2} \omega^{2}\right), & j=0 \\
1, & \omega=0
\end{array}\right.
$$

then, using the fact that the $I_{k}$ 's given $B$ are independent, we have the characteristic function for the total interference term $I$, given $B$, as

$$
\Phi_{I \mid B}(\omega)=\prod_{k=2}^{K} \Phi_{I_{k} \mid B}(\omega)
$$

Let, $\xi|B=I| B+n_{1}$, where $n_{1}$ is a Gaussian random variable representing the background noise, $I \mid B$ is the total other-user interference given $B$, and $\xi \mid B$ is the total disturbance given $B$. Since the other-user interference and background noise are independent, we have

$$
\begin{aligned}
\Phi_{\xi \mid B}(\omega) & =\Phi_{I \mid B}(\omega) \Phi_{n_{1}}(\omega) \\
& =\Phi_{n_{1}}(\omega)-\left[1-\Phi_{I \mid B}(\omega)\right] \Phi_{n_{1}}(\omega)
\end{aligned}
$$

We use the Fourier inversion formula for the real integral to find the distribution function of $\xi \mid B, F_{\xi \mid B}(\cdot)$ which is to be used to calculate the BER, as

$$
F_{\xi \mid B}(\xi)=\frac{1}{2}+\frac{1}{\pi} \int_{0}^{+\infty} \frac{\Phi_{I \mid B}(\omega)}{\omega} \sin (\xi \omega) d \omega
$$

The conditional BER for our target user can be expressed, by symmetry, as

$$
\begin{aligned}
P_{e \mid A_{1}, B} & =P\left\{\xi<-A_{1} N\right\} \\
& =1-F_{\xi}\left(A_{1} N\right) \\
& =\frac{1}{2}-\frac{1}{\pi} \int_{0}^{+\infty} \frac{\sin \left(A_{1} N\right)}{\omega} \Phi_{\xi \mid B}(\omega) d \omega \\
& =Q\left(\frac{A_{1} N}{\sigma_{n 1}}\right)+\frac{1}{\pi} \int_{0}^{+\infty} \frac{\sin \left(A_{1} N \omega\right)}{\omega} \times\left[1-\Phi_{I \mid B}(\omega)\right] \Phi_{n 1}(\omega) d \omega
\end{aligned}
$$


Averaging over the pdf of $A_{1}$, and using the integral identity

$$
\int_{0}^{+\infty} \sin (k x) x e^{-x^{2} / 2} d x=\sqrt{\frac{\pi}{2}} k e^{-k^{2} / 2}
$$

we have,

$$
\begin{aligned}
P_{e \mid B}= & \frac{1}{2}\left[1-\frac{N}{\sqrt{\sigma_{n 1}^{2}+N^{2}}}\right]+\frac{N}{\sqrt{2 \pi}} \int_{0}^{+\infty}\left[1-\Phi_{I \mid B}(\omega)\right] \Phi_{n 1}(\omega) \\
& \times \exp \left\{-\frac{1}{2} \omega^{2} N^{2}\right\} d \omega
\end{aligned}
$$

which finally yields,

$$
P_{e \mid B}=\frac{1}{2}-\frac{N}{\sqrt{2 \pi}} \int_{0}^{+\infty} \Phi_{I \mid B}(\omega) \Phi_{n 1}(\omega) \exp \left\{-\frac{1}{2} \omega^{2} N^{2}\right\} d \omega
$$

where the characteristic function of noise is given by

$$
\Phi_{n 1}(\omega)=e^{-\left\{v^{2}(1-\varepsilon)^{2}+k^{3} v^{2} \varepsilon^{2}\right\} \omega^{2}}
$$

\section{RobUst MUltiUSER DETECTION}

The basic idea of $M$-estimator based multiuser detection is to detect the symbols in (1) by first estimating the vector $\theta(i)$, and then extracting symbol estimates from these continuous estimates [1] \& [2]. The required estimates of $\theta(i)$ are obtained by using $M$-estimators proposed by Huber [3]. $M$ estimators minimize a sum of function $\rho$ (.) of the residuals

$$
\begin{aligned}
\hat{\theta}(i)=\underset{\theta(i) \in C^{L}}{\arg \min } \sum_{n=1}^{N}\{\rho & \left.\Re\left\{y_{n}(i)-\sum_{k=1}^{L}[A]_{n k} \theta_{k}(i)\right\}\right) \\
& \left.+\rho\left(\mathfrak{I}\left\{y_{n}(i)-\sum_{k=1}^{L}[A]_{n k} \theta_{k}(i)\right\}\right)\right\}
\end{aligned}
$$

where $y_{n}(i)$ and $\theta_{k}(i)$ are the $n$th and the $k$ th element of the vectors $\boldsymbol{y}(i)$ and $\theta(i)$, respectively, $[\boldsymbol{A}]_{n k}$ is the $n, k$ th element of the matrix $\boldsymbol{A}, \mathfrak{I}$ denotes imaginary part, and $\rho$ is a symmetric, positive-definite function with a unique minimum at zero, and is chosen to be less increasing than square. Suppose that $\rho$ has a derivative $\left(\psi=\rho^{\prime}\right)$, then the solution to (21) satisfies the implicit equation

$$
\begin{aligned}
\sum_{n=1}^{N}\left\{\psi\left(\Re\left\{y_{n}(i)-\sum_{k=1}^{L}[A]_{n k} \theta_{k}(i)\right\}\right)\right. \\
\left.+\psi\left(\mathfrak{I}\left\{y_{n}(i)-\sum_{k=1}^{L}[A]_{n k} \theta_{k}(i)\right\}\right)\right\}[A]_{n k}=0, k=1, \ldots, L
\end{aligned}
$$

In an impulsive noise environment, a more efficient estimator can be obtained by considering a less sensitive function $\rho($.$) of the residuals. Hence, the following penalty$ function $\rho($.$) and the corresponding influence functions \psi($. are proposed (also see Figure 1)

$$
\rho_{\text {PROPOSED }}(x)=\left\{\begin{array}{c}
\frac{x^{2}}{2}, \text { for } \quad|x| \leq a \\
\frac{a^{2}}{2}-a|x|, \text { for } \quad a<|x| \leq b \\
-\frac{a b}{2} \exp \left(1-\frac{x^{2}}{b^{2}}\right)+d, \text { for } \quad|x|>b
\end{array}\right.
$$

and

$$
\Psi_{\text {PROPOSED }}(x)=\left\{\begin{array}{c}
x, \text { for } \quad|x| \leq a \\
a \operatorname{sgn}(x), \text { for } \quad a<|x| \leq b \\
\frac{a}{b} x \exp \left(1-\frac{x^{2}}{b^{2}}\right), \text { for } \quad|x|>b
\end{array}\right.
$$

where $d$ is a constant.

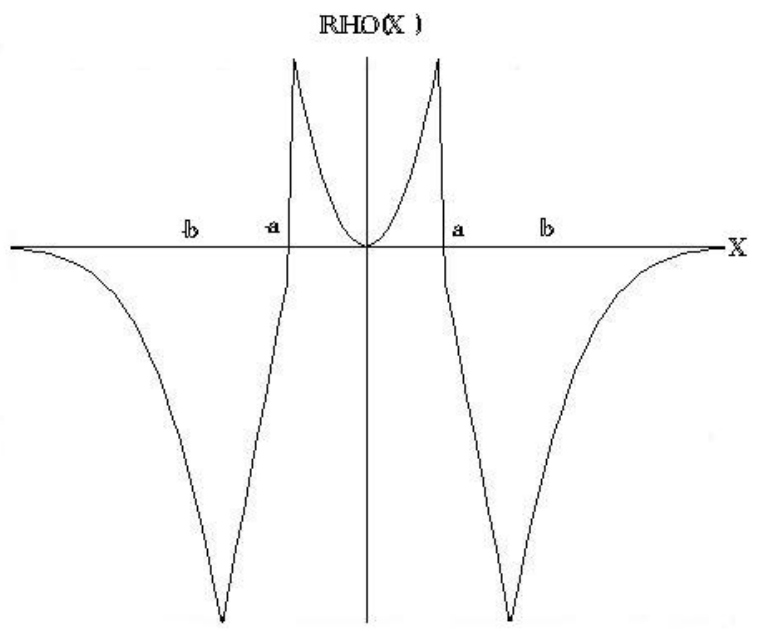

(a) 


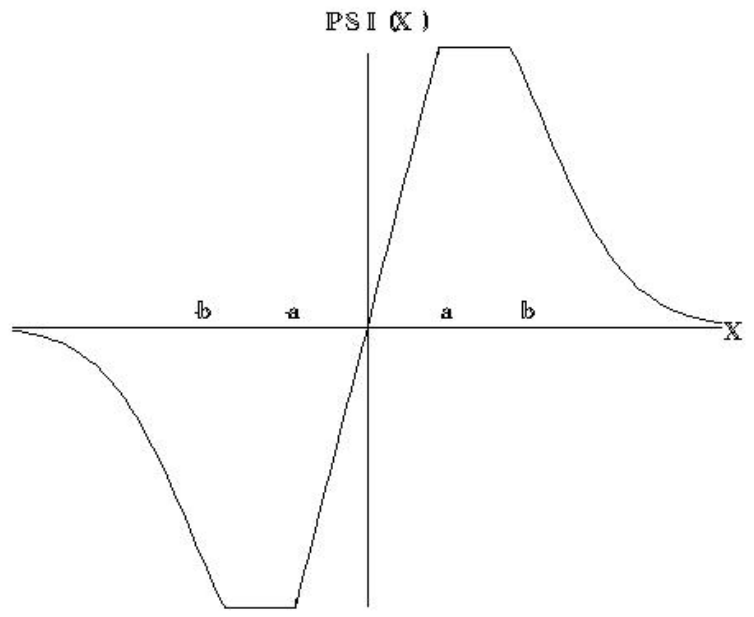

(b)

Figure 1. (a) Penalty function and (b) influence functions of the proposed estimator

\section{Simulation Results}

In simulations, a CDMA system with 6 users, in which the spreading sequence of each user is a shifted version of $\mathrm{m}$ sequence, is considered. The fading channel is modeled (having a perfect knowledge of the channel coefficients $g_{l}(i)$ $(l=1,2, \ldots, L))$ as [5]. The performance of the proposed detector as a function of signal-to-noise ratio (SNR) in asynchronous flat-fading non-Gaussian channel (with $\varepsilon=0.1$ and $0.01, \kappa=$ 100) for $N=127$ is shown in Fig. 2 and 3. The bit rate, the pole radius, and the spectral peak frequency have been fixed at $1 / T=10 \mathrm{~Kb} / \mathrm{s}, r_{d}=0.998$, and $f_{p}=80 \mathrm{~Hz}$.

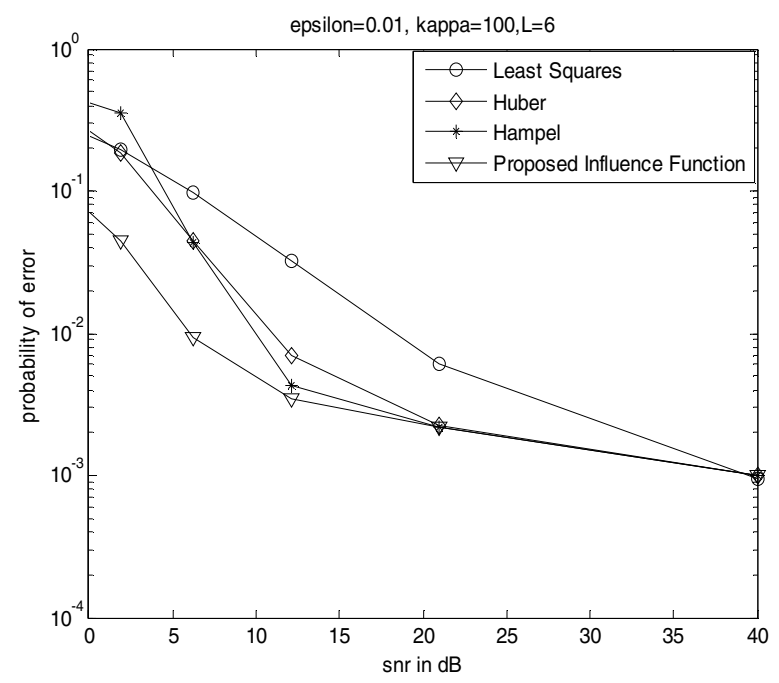

Figure 2. Probability of error versus SNR for user 1 for the considered detectors in asynchronous flat-fading CDMA channel with non-Gaussian noise. $N=127$.

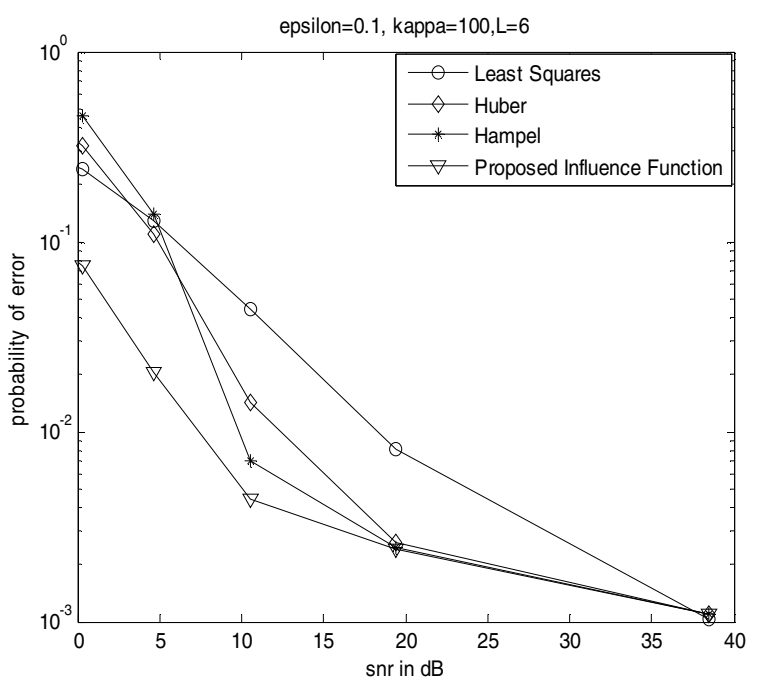

Figure 3. Probability of error versus SNR for user 1 for the considered detectors in asynchronous flat-fading CDMA channel with non-Gaussian noise. $N=127$.

These Simulation results show that the proposed detector with the proposed influence function outperforms the linear decorrelating detector and minimax detectors (with Huber and Hampel estimators) in asynchronous flat-fading non-Gaussian channels.

\section{CONCLUSIONS}

In this paper, a new closed-form expression is derived for computing the average BER in asynchronous flat-fading nonGaussian channels using the characteristic function method. Further a new $M$-estimator based multiuser detection technique is proposed that is seen to significantly outperform linear decorrelating detector and minimax detectors (with Huber and Hampel $M$-estimators) in asynchronous flat-fading CDMA channels with impulsive noise with little attendant increase in the computational complexity.

\section{REFERENCES}

[1] X. Wang and H. V. Poor, "Robust multiuser detection in non-Gaussian channels," IEEE Trans. Signal Processing, vol. 47, 289-305, Feb. 1999.

[2] T.Anil Kumar, and K.Deergha Rao, "Improved robust techniques for multiuser detection in non- Gaussian channels," Circuits Systems and Signal Processing J., Vol. 25, No. 4, 2006.

[3] P. J. Huber, Robust Statistics. New York: Wiley, 1981.

[4] F. R. Hampel et al., Robust Statistics: The Approach Based on Influence Functions. New York: Wiley, 1986.

[5] Z. Zvonar and D. Brady, "Multiuser detection in single-path fading channels," IEEE Trans. Commun., vol. 42, pp. 1729-1739, Feb.-Apr. 1994.

[6] J. Cheng and N. C. Beaulieu, "Accurate DS-CDMA Bit-Error Probability Calculation in Rayleigh Fading," IEEE Trans. Wireless Commun., vol. 1, pp. 3-15, Jan. 2002. 